\title{
The UKIRT wide-field camera
}

\author{
M. Casali ${ }^{1}$, A. Adamson ${ }^{3}$, C. Alves de Oliveira ${ }^{1}$, O. Almaini ${ }^{7}$, K. Burch ${ }^{2}$, T. Chuter ${ }^{3}$, J. Elliot ${ }^{2}$, M. Folger ${ }^{2}$,

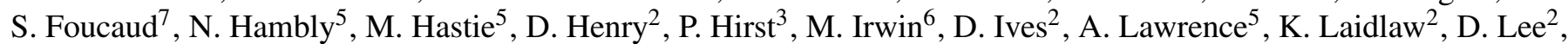 \\ J. Lewis ${ }^{6}$, D. Lunney ${ }^{2}$, S. McLay², D. Montgomery ${ }^{2}$, A. Pickup ${ }^{2}$, M. Read ${ }^{5}$, N. Rees ${ }^{3}$, I. Robson ${ }^{2}$, K. Sekiguchi ${ }^{8}$, \\ A. Vick ${ }^{2}$, S. Warren ${ }^{4}$, and B. Woodward ${ }^{2}$ \\ ${ }^{1}$ European Southern Observatory, Karl-Schwarzschild Str.2, 85748, Garching bei Muenchen, Germany \\ e-mail: mcasali@eso.org \\ 2 UK Astronomy Technology Centre, Blackford Hill, Edinburgh, EH9 3HJ, UK \\ 3 Joint Astronomy Centre, 660 N. Aohoku Place, University Park, Hilo, Hawaii, 96720, USA \\ 4 Astrophysics Group, Imperial College London, Blackett Laboratory, Prince Consort Rd, London, SW7 2AZ, UK \\ 5 Institute for Astronomy, University of Edinburgh, Blackford Hill, Edinburgh, EH9 3HJ, UK \\ ${ }^{6}$ Institute of Astronomy, University of Cambridge, Madingley Rd, Cambridge, CB3 OHA, UK \\ 7 School of Physics and Astronomy, University of Nottingham, University Park, Nottingham, NG7 2RD, UK \\ 8 Subaru telescope, National Astronomical Observatory of Japan, 650 N.Aohoku Place, Hilo, Hawaii 96720, USA
}

Received 6 October 2006 / Accepted 20 February 2007

\section{ABSTRACT}

\begin{abstract}
Context. The infrared wide-field camera (WFCAM) is now in operation on the $3.8 \mathrm{~m}$ UK Infrared Telescope on Mauna Kea. WFCAM currently has the fastest survey speed of any infrared camera in the world, and combined with generous allocations of telescope time, will produce deep maps of the sky from $Z$ to $K$ band. The data from a set of public surveys, known as UKIDSS, will be initially available to astronomers in ESO member states, and later to the world.

Aims. In order to maximise survey speed, the WFCAM field of view was required to be as large as possible while incorporating conventional infrared-instrument design features such as a cold re-imaged pupil stop and cryogenic optics and mechanisms.

Methods. The solution adopted was to build a cryogenic Schmidt-type camera, mounted forward of the primary mirror, which illuminates a very large $0.9^{\circ}$ diameter focal plane, containing four $2 \mathrm{k} \times 2 \mathrm{k} \mathrm{HgCdTe}$ Rockwell detectors.

Results. Following several commissioning periods during which the camera, focal plane and telescope optical axes were successfully co-aligned, WFCAM now operates close to specifications, regularly achieving $0.7^{\prime \prime}$ FWHM images over the full field. Projects which already report excellent results include the detection of variability in young stellar clusters, as well as preliminary deep IR imaging of the Subaru and XMM-Newton deep field.
\end{abstract}

Key words. instrumentation: miscellaneous - infrared: general

\section{Introduction}

The worldwide development of $8 \mathrm{~m}$ class telescopes as new facilities for conducting ground-based optical and IR astronomy raises questions regarding the future use and scientific competitiveness of older existing 4-m class and smaller telescopes. Although $8 \mathrm{~m}$ telescopes will always outperform smaller telescopes for the study of single distinct objects, this is not true in the case of wide area imaging surveys. In this case the speed with which a survey covers a given area of sky to a given depth is, to first order, proportional to the product of aperture area and field of view (étendue). Since it is generally easier to achieve wide fields of view on small telescopes, their étendue may exceed that of larger facilities and so these telescopes are capable of cuttingedge scientific survey projects. The Schmidt telescopes are the best examples of this. The Palomar, UK and ESO Schmidt surveys have made major contributions to astronomy, and provided countless targets for study by 2 and $4 \mathrm{~m}$ telescopes over the last 40 years, despite having only $1-\mathrm{m}$ class apertures. More recently, the Sloan Digital Sky Survey (York et al. 2000) has continued this work, while 2MASS (Skrutskie et al. 2006) has for the first time extended large-area moderately-deep surveys into the infrared. As general-purpose telescopes become larger, however, surveys need to go deeper to provide lists of suitable targets for study. So just as the $1.2 \mathrm{~m}$ Schmidt telescopes provided targets for study by 2 and $4 \mathrm{~m}$ telescopes, the development of survey capabilities on $2 \mathrm{~m}$ and $4 \mathrm{~m}$ class telescopes becomes essential to feed new $8 \mathrm{~m}$ projects.

WFCAM was conceived in this spirit as a wide-field imager for the $3.8 \mathrm{~m}$ UK Infrared Telescope on Mauna Kea. The intention was to provide astronomers with unprecedented deep and large-scale infrared imaging. 2MASS has been a ground breaking project, but its relatively bright limiting magnitude $(K=$ 14.3) does not match the typical spectroscopic limits on $8 \mathrm{~m}$ telescopes (e.g. $K=17$ on ISAAC-VLT at medium resolution in $1 \mathrm{~h}$ ). WFCAM on UKIRT has a number of enhancements which means it is more than $100 \times$ faster at surveying than 2 MASS. The $3.8 \mathrm{~m}$ primary mirror gives a factor of 8.5 increase in survey speed, the larger single-exposure field area gives a factor of 3.4, and better image quality and smaller pixel size another factor of 4 . In addition the cold telescope temperatures on Mauna Kea give further improvements in $K$ band. WFCAM is therefore able to explore very large areas of the faint high-redshift universe for the first time in the infrared. Such a large jump in capabilities also means that exciting science can be obtained from the WFCAM survey database alone, without requiring any 


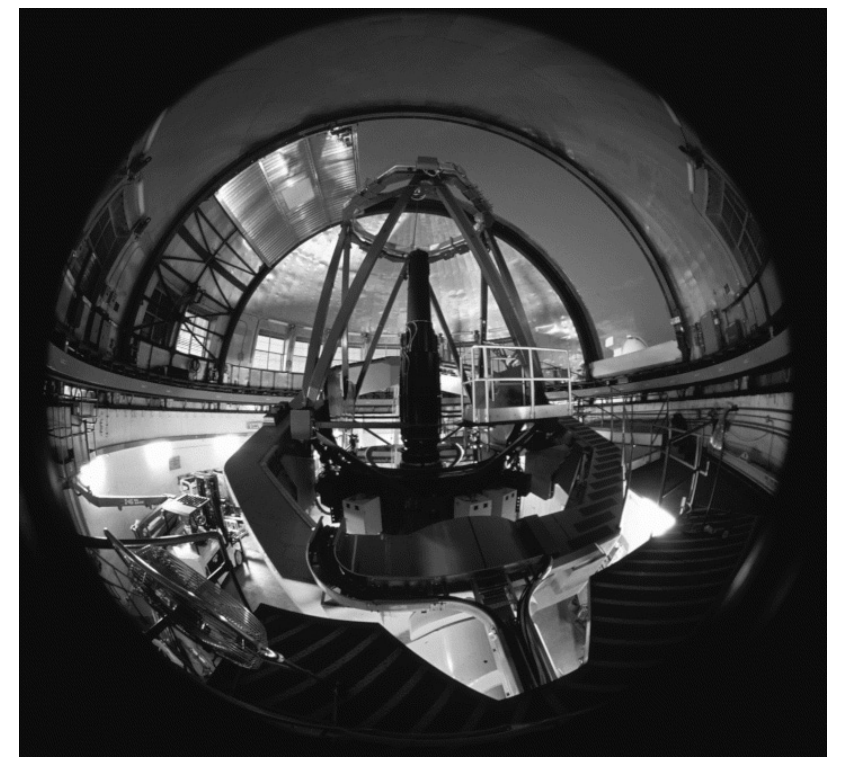

Fig. 1. WFCAM on the $3.8 \mathrm{~m}$ UK Infrared Telescope. WFCAM is the tall cylindrical structure mounted above the primary mirror. Photo courtesy of P. Hirst.

immediate follow-up on larger facilities. An instrument of similar power, WIRCAM, has also been constructed for the CanadaFrance-Hawaii Telescope (Puget et al. 2004)

The WFCAM IR camera project began at the UK Astronomy Technology Centre in Edinburgh, following Particle Physics and Astronomy Research Council approval in 1999. The completed camera was shipped to the Joint Astronomy Centre in Hawaii in June 2004, and phase 1 of commissioning ended in October 2004. Figure 1 shows the camera on the telescope. Three of WFCAMs four large-format detectors were provided by the SUBARU telescope in exchange for UKIRT telescope time.

WFCAM is being used for both public surveys and individual principal investigator projects. The public data will consist of five hierarchical surveys, designed by a consortium of UK astronomers (Lawrence et al. 2006) and known collectively as UKIDSS (UKIRT Infrared Deep Sky Survey). The data product releases will be made public simultaneously to all astronomers in ESO member states via the WFCAM Science Archive (http://surveys.roe.ac.uk/wsa), with a general world release 18 months later. An Early Data Release (EDR), amounting to $1 \%$ of the expected final UKIDSS data volume, was made available in February 2006 (Dye et al. 2006), while the first major data release (DR1) occurred in July 2006.

The purpose of this paper is to provide a general description of the WFCAM instrument for astronomers who will be using UKIDSS data, and for instrumentation scientists. A great deal of engineering detail has therefore deliberately been left out, though some extended discussion on novel aspects of the design have been included where appropriate. Specifications are discussed in Sect. 2, and the general design features presented in Sect. 3. WFCAM operational aspects are discussed in Sect. 4. Finally, performance results are given in Sect. 5.

This is one of a series of papers describing WFCAM and UKIDSS. These describe the UKIDSS surveys (Lawrence et al. 2006), calibration (Hewett et al. 2006), pipeline data reduction (Irwin et al. 2006), and archiving (Hambly et al. 2006). Publications describing WFCAM

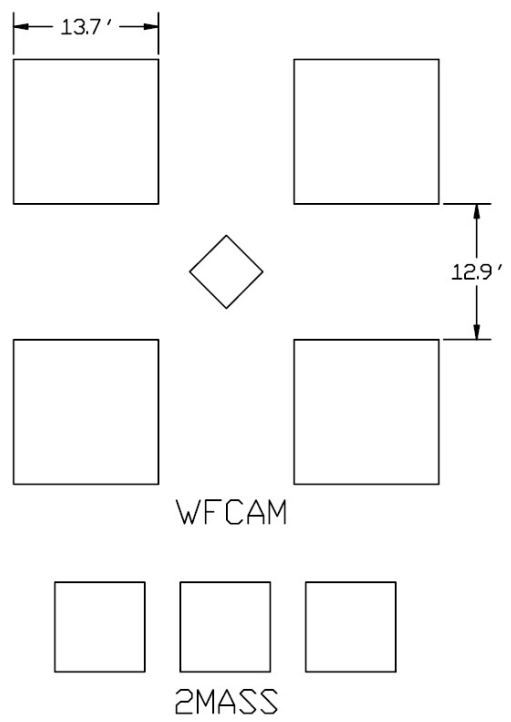

Fig. 2. WFCAM focal plane on the sky. Dimensions of the field of view are shown along with the 2MASS field (3 simultaneous bands) for comparison. The central diamond shape is the autoguider CCD field.

sub-systems and commissioning in more engineering detail can be found in Henry et al. (2003) and Hirst et al. (2006).

\section{WFCAM specifications}

WFCAM was designed from the beginning to maximise survey speed, defined as the rate at which the sky can be surveyed to a given depth. The focal plane specification was central to achieving this, so a set of three focal plane requirements were set early as key specifications driving most subsequent optomechanical, electronic and software design.

- WFCAM should have four $2 \mathrm{k} \times 2 \mathrm{k}$ IR detectors.

- The WFCAM pixel scale should be 0.4 arcsec on the sky. This choice followed numerical simulations using typical UKIRT point spread functions. For a fixed total number of pixels (set largely by cost) and background photon-noise limited operation, survey speed is optimum when pixel size is of the order of the average point-spread function (PSF) size. Although at the time of design UKIRT median seeing was 0.6 arcsec, a pixel size this big was not achievable in the optical design, requiring too fast a final f-ratio, and a pixel size of 0.4 arcsec was selected. Ultimately, the combination of seeing, optical aberrations, and misalignments produce a final $K$-band median image quality of 0.7 arcsec. So the pixel scale slightly undersamples the PSF on average. A microstepping observing mode is available to improve the sampling by stepping in half or third-pixel increments.

- The detectors should be separated by $94 \%$ of their active area, in a square pattern. This is because the available detector arrays at the time had substantial packaging around the active area. When detectors cannot be butted together, it is usually more efficient to space them by slightly less than a full detector size (to allow overlap), provided the optical design can deal with the larger field of view that results.

The focal plane layout is shown in Fig. 2. The required field $\left(0.9^{\circ}\right.$ diameter $)$ is extremely large by IR imaging standards on $4 \mathrm{~m}$ telescopes, and is compared with the 2MASS field. Note that the latter imaged three simultaneous bands, in $J, H$ and $K$, hence three detector fields are shown. 
Table 1. WFCAM filter specifications at operating temperature.

\begin{tabular}{ccccc}
\hline \hline Filter & $\begin{array}{c}50 \% \\
\text { cut-on }\end{array}$ & $\begin{array}{c}50 \% \\
\text { cut-off }\end{array}$ & $\begin{array}{c}\text { Average } \\
\text { transmission }\end{array}$ & $\begin{array}{c}\text { Typical } \\
\text { sky/Tel } \\
\text { background } \\
\text { elec/s/pixel }\end{array}$ \\
\hline$Z$ & $\mu \mathrm{m}$ & $\mu \mathrm{m}$ & & 50 \\
$Y$ & 0.83 & 0.925 & 0.94 & 80 \\
$J$ & 1.17 & 1.07 & 0.80 & 300 \\
$H$ & 1.49 & 1.73 & 0.82 & 2300 \\
$K$ & 2.03 & 2.37 & 0.86 & 2500 \\
$\mathrm{~S} 1$ & 2.111 & 2.132 & 0.78 & 100 \\
$\mathrm{Br} \gamma$ & 2.155 & 2.177 & 0.85 & 180 \\
\hline
\end{tabular}

The filter choices shown in Table 1 were relatively conventional, consisting of broad band JHK filters on the Mauna Kea photometric system (Tokunaga et al. 2002). Note that the WFCAM $K$ is the full broadband filter, not a short $K$. Narrowband filters provide imaging capability at rest wavelengths for the 1-0 S(1) line of molecular hydrogen and the Brackett $\gamma$ recombination line of hydrogen. A detailed study of the WFCAM photometric system is presented in Hewett et al. (2006).

The $Y$ filter is somewhat unusual. It occupies the clean wavelength range between the atmospheric absorption bands at 0.95 and $1.14 \mu \mathrm{m}$. The bandpass is similar to, but slightly narrower than, the $Y$ bandpass of Hillenbrand et al. (2002). The longwavelength cut-off of the WFCAM $Y$ filter is at a significantly bluer wavelength, and largely avoids the strong sky emission lines near $1.1 \mu \mathrm{m}$. Not only does this reduce the sky background, but it improves the $Y-J$ colour discrimination between highredshift quasars and $\mathrm{T}$ dwarfs.

The $Z$ filter inclusion followed encouraging laboratory quantum efficiency measurements at $800 \mathrm{~nm}$ on the first Hawaii-2 engineering device received, which suggested values above $30 \%$ were likely. The inclusion of a $Z$ band gives WFCAM the maximum possible wavelength baseline. The bandpass of the $Z$ filter was designed to provide a reasonable match to the effective wavelength of the SDSS $z^{\prime}$ bandpass, but with a rectangular profile, and avoiding the atmospheric absorption band near $0.95 \mu \mathrm{m}$. The effective wavelengths of the WFCAM $Z$ and SDSS $z^{\prime}$ bandpasses are $0.876 \mu \mathrm{m}$ and $0.887 \mu \mathrm{m}$, respectively.

Table 2 gives a summary of the other main WFCAM characteristics. This is of course only a representative subset of the full list of engineering specifications.

The WFCAM image quality requirement was one of the most challenging aspects of the project. The achievement of good image quality over a large field, in a design with a field lens/cold pupil stop, required careful tolerancing, manufacture, assembly, and instrument alignment. The alignment process was not helped by the fact that the intermediate focus from the new M2 was highly aberrated as a result of the optimised overall design, making testing of separate sub-assemblies difficult. It was therefore decided to design WFCAM such that the final optical image quality resulted from achievable optical surface and metal-machining tolerances, and careful assembly in the lab, without relying on extensive sub-system testing. In the end this was achieved in all but one respect; the practical limitations in setting the focal plane orthogonal to the optical axis meant that this had to be done with an iterative procedure on the telescope. After several attempts the focal plane was set correctly.

An interesting aspect of the image quality specification is its definition in terms of both an $80 \%$ encircled energy and a modulation transfer function (MTF). The latter, which measures the image contrast as a function of spatial frequency, is common in commercial optics manufacture but is less used in astronomy where full-width at half maximum $(F W H M)$ of the point spread function is usually adopted. The MTF has the advantage of giving a much better idea of the real achieved image contrast compared to $F W H M$. For example, consider a Gaussian and an exponential function, both with an identical FWHM of $0.7^{\prime \prime}$. At a spatial frequency of 0.88 cycles per arcsec, the MTF (image contrast) of the Gaussian has dropped to 0.5, while the MTF of the exponential is 0.19; the Gaussian has more than twice the contrast at this spatial frequency, a fact that is lost in the FWHM approach. The MTF specification adopted is shown in Table 2.

\section{Instrument description}

\subsection{Optical design}

The ambitious focal plane requirements described in Sect. 2 had far-reaching implications for the optical design. For a $3.8 \mathrm{~m}$ telescope, these requirements imply a final $f$-ratio of $f / 2.4$, and a field of view of 0.9 degrees in diameter. Approximately a year of investigation of various designs was done before the current solution was adopted as the baseline. The reasons for rejecting all the various alternative designs are not discussed here. However it is worth pointing out that two of the more obvious designs can be easily ruled out. Firstly, use of the prime focus, in principle ideal because the UKIRT primary is $f / 2.5$, was not possible due to UKIRTs small dome and near-zero clearance for a prime focus instrument. Secondly, placing the instrument at a classical Cassegrain focus would have resulted in very large optics to achieve the final $f$-ratio required and would have required a complete dismantling of the normal Cassegrain instrument support unit each time WFCAM was installed. The adopted design achieves very good image quality over a large field, while minimizing disruption to the telescope.

The WFCAM optical design is shown in two parts in Fig. 3. One may think of the design, broadly, as a large cryogenic quasiSchmidt camera with finite conjugate foci. It may be described as follows. A new M2 for the telescope provides an $f / 9$ intermediate focus $5.7 \mathrm{~m}$ above M1, UKIRTs parabolic primary mirror. A simple bi-convex field lens at this point (L1) re-images M1 at a cold, slightly-undersized $396 \mathrm{~mm}$ diameter pupil stop S inside the cryostat, necessary for thermal straylight control at $K$ band. With fused silica as the only practical IR optical material available in such large sizes, any significant optical power must be in reflective surfaces to avoid chromatic aberrations, and so the final f-conversion from 9 to 2.4 is achieved with M3, a Zerodur ellipsoidal mirror with double-arch rear shaping mounted deep within the vacuum vessel (V). M3 is mounted on three flexures which allow differential contraction with respect to its aluminium mounting plate when cooled to cryogenic temperatures, while supporting the mirror to the required flexure tolerances as the telescope changes attitude during observing. M3 is one of the largest mirrors ever used cryogenically for astronomy; at $802 \mathrm{~mm}$ it is only slightly smaller than the Spitzer $850 \mathrm{~mm}$ primary mirror.

Since the new M2 is mismatched with M1, large spherical and off-axis aberrations result at the intermediate focus near L1. These are mainly corrected by the corrector plate L2, just inside the window W. L2 has a single, aspheric surface and is a crucial component for the satisfactory optical performance of WFCAM.

The focal plane assembly (FP) involves three optical components. A filter plate contains four $60 \mathrm{~mm}$ square filters (one per detector) used for wavelength selection. A field flattening lens, 
Table 2. Main WFCAM specifications.

\begin{tabular}{lll}
\hline \hline Item & Specification & Units and notes \\
\hline Detectors & 4 Rockwell Hawaii-2 & PACE HgCdTe devices operating from 0.8 to 2.5 $\mu \mathrm{m}$ \\
Focal plane non-coplanarity & 50 & Microns. Peak-to-valley \\
Signal digitization & 16 & Bits \\
Number of parallel channels & $128(32$ per detector) & In parallel, with synchronized clocks \\
Available read modes & correlated double sampling, & \\
& fowler sampling, & \\
& up-the-slope sampling & \\
Pixel scale & $0.40 \pm 0.01$ & Arcsec \\
Single-shot field of view & 0.21 & Square degrees \\
2×2 tile field of view & 0.79 & Square degrees \\
Image Quality(MTF) & $>0.5$ & At a spatial frequency of 1.2 cycles per arcsec \\
Image Quality(encircled energy) & $>80 \%$ & Within a diameter of 1.2 arcsec \\
Autoguider CCD & E2V $47-10$ & Backthinned non-inverted mode \\
& & $1056 \times 1027$ pixels \\
Autoguider window sample rate & $20-100$ & $\mathrm{~Hz}$ \\
\hline
\end{tabular}
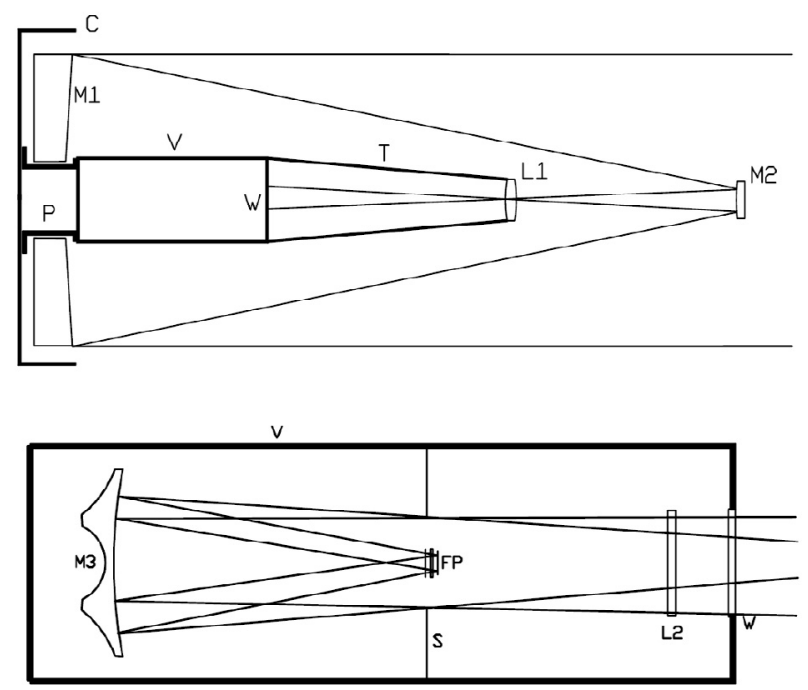

Fig. 3. WFCAM optical layout showing the overall design on the telescope at top, with details of the cryostat optics below.

cut square, serves to give a flat field over the detector plane. Thirdly, the square detector box itself holds the four detectors coplanar, incorporates their four PCBs, and encloses a shielded feed-through for the autoguider signals. Since the focal plane assembly directly vignettes the incoming beam, it was also designed to be as compact as possible, while allowing mechanical adjustments of detector position and coplanarity.

The entire optical design was optimised for best average image quality over the whole field from $J$ to $K$ bands, and system tolerancing was checked through Monte-Carlo analyses. The resulting design gives near diffraction limited performance at $K$-band over the whole field of view.

\subsection{Thermal design}

A liquid nitrogen pre-cooling line which feeds an internal annular can is used for initial rapid instrument cooling. During regular operation the entire cryostat is cooled by a single, two-stage Gifford-McMahon CTI 1050 Cryocooler. This is sufficient given the modest temperature requirements of the instrument resulting from the 2.5 micron detector cutoff and $70 \mathrm{~W}$ thermal load.

A key element of the thermal design is the placement of the aspheric corrector (L2) inside the cryostat, close to the window (W), and allowing it to thermally float using fibreglass supports. Not only does this ensure an even temperature distribution over L2, but allows it to act as a floating radiation shield, effectively halving the radiation load from the window and helping to keep it warm. As a further precaution against condensation, the field lens tower $(\mathrm{T})$ is constantly flushed with a dry nitrogen supply. These features were incorporated to circumvent any condensation problems on the window, and indeed none has been seen during operation on the telescope.

M3 is a large cryogenic Zerodur mirror. In fact this mirror does not need to be particularly cold, but its position deep in the vacuum vessel means it naturally equilibrates at approximately $120 \mathrm{~K}$. In order to ensure a uniform temperature distribution, the mirror design allows it to cool radiatively; three A-frame flexure supports made of G10 fibreglass provide sufficient conductive isolation. After a few days of cooling, any remaining changes in mirror temperature predominantly cause only a focus shift, with only a small amount of trefoil distortion due to cooling through the fibreglass supports.

The thermal contribution from the cryostat and telescope is an important issue for instruments operating in $K$ band or longer wavelengths. It is particularly an issue for WFCAM because of its peculiar optical layout in which pixels are in principle able to see the outside of the vacuum vessel reflected in the secondary mirror. To deal with this an extra cold circular obscuring baffle was placed in each filter paddle along with filters in $K$ band. There are no such baffles for $H$ band and shorter wavelengths. Since we have no pupil imaging mode, we rely on measurements of the sky background for confirmation that all sources of warm emission have been properly baffled. Measurements of background brightnesses, shown in Table 1, are consistent with typical values on UKIRT for other simple imagers, so we conclude that all significant sources of thermal emission in the WFCAM beam have been adequately baffled.

\subsection{Detectors and focal plane}

The four infrared detectors used in WFCAM are $\mathrm{HgCdTe}$ Rockwell Hawaii-2 devices. These are in a $2048 \times 2048$ format with 18 micron pixels and were the first generation detectors in this format from Rockwell Scientific (now Teledyne). The detection layer uses the Rockwell PACE (liquid-phase epitaxy) process in manufacturing, with the $\mathrm{HgCdTe}$ layer deposited on a saphire crystal substrate. Each $2 \mathrm{k} \times 2 \mathrm{k}$ detector consists of four identical $1 \mathrm{k} \times 1 \mathrm{k}$ quadrants, each with eight $128 \times 1 \mathrm{k}$ readout 
Table 3. WFCAM performance.

\begin{tabular}{|c|c|c|}
\hline$\overline{\text { Item }}$ & Specification & Units and notes \\
\hline Average detector $\mathrm{QE}$ & $0.54,0.62,0.61$ & In $J, H, K$, and averaged over 4 devices \\
\hline Focal plane Coplanarity & 50 & $\mu \mathrm{m}$ peak-to-valley over 4 devices \\
\hline IR Pixel clock rate & 187 & $\mathrm{kHz}$ \\
\hline Focal plane readout time & 0.7 & Seconds. Includes extra reset for stability \\
\hline Read noise & $<30$ & Electrons in correlated double sample mode \\
\hline Median delivered image quality & 0.7 & Arcseconds. $K$-band $F W H M$. Near zenith \\
\hline Best delivered image quality & 0.55 & Arcseconds. $K$-band $F W H M$. Near zenith \\
\hline Astrometric errors & $<0.1$ & $\begin{array}{l}\text { Arcseconds over full field of view, after } \\
\text { correction for field distortion }\end{array}$ \\
\hline Field distortion & $0.3 \%$ & Centre-to-corner change in plate scale \\
\hline Systematic photometric errors & $<0.02$ & Magnitudes. Over the field of view \\
\hline Total system throughput in $\mathrm{Z}, \mathrm{Y}, \mathrm{J}, \mathrm{H}, \mathrm{K}$ & $0.14,0.16,0.20,0.28,0.29$ & $\begin{array}{l}\text { including telescope, filter and detector } \mathrm{QE} \\
\text { Relative to a perfect telescope and instrument } \\
\text { of } 10.5 \text { sq. m collecting area }\end{array}$ \\
\hline Autoguider magnitude limit & 17 & $I$ band at $40 \mathrm{~Hz}$. No moon \\
\hline WFCAM average observing efficiency & 0.55 & (time spent integrating)/(elapsed time) \\
\hline
\end{tabular}

strips. The later version ( $2 \mathrm{RG}$ ) offers substantial performance improvements and new features such as on-chip interlaced readout of a guide window, but was unavailable in time for WFCAM. The measured quantum efficiencies of the science detectors are listed in Table 3.

A recent report by Finger et al. (2006) has shown that the traditional approach of using photon-noise transfer curves to calculate the system gain (photons per data number) suffers from a systematic error due to stray inter-pixel capacitance. This results in an overestimate of the photons per data number, and a subsequent overestimate of detector quantum efficiencies. The error varies from device to device and for the Hawaii- 2 devices this appears to be of order $20 \%$. The quantum efficiencies shown in Table 3 have been corrected for this and are therefore $20 \%$ less than the numbers from the manufacturer.

Although it is known that the quantum efficiency drops off rapidly below $800 \mathrm{~nm}$, direct laboratory quantum efficiency measurements for the science devices at $Z$ and $Y$ bands were not available from the manufacturer. Tests were done, therefore, on an early engineering device and values above $30 \%$ at $800 \mathrm{~nm}$ were determined, and it was on the basis of these results that the $Z$ filter was procured. Observations of standard stars during commissioning imply quantum efficiencies of order $40-50 \%$ in the science-quality devices in $Z$ band.

The fast final $f$-ratio required a coplanarity error over the whole focal plane of 50 microns peak-to-valley. Since the detectors are each mounted on a ceramic carrier, which in turn is inserted into a plastic ZIF socket in the standard manner recommended by Rockwell, only low mechanical precision is possible by design. So the deviation from coplanarity had to be measured, and appropriate shims adjusted. Since the thickness of each sapphire substrate was not known a priori, it was decided to measure this directly using the IR detectors themselves. This was done using a projector and two-spot Hartmann mask, with the detectors cold and operating inside a test cryostat with large window. After some tweaking of the system hardware and software we were able to obtain a precision of $4-5 \mu \mathrm{m}$, and the final coplanarity specification was finally achieved with this technique in 2004.

\subsection{Cryomechanisms}

Many instruments do not incorporate a separate focussing stage since focussing is accomplished by piston motions of the telescope secondary mirror. However, the WFCAM optics rely on a careful balancing of aberrations which requires the optics to be at known positions. Since manufacturing tolerances meant that the telescope M2 might need to be moved a substantial amount from its design position to achieve focus, thereby introducing spherical aberration, it was decided to incorporate an internal mechanical focussing stage as well. This decision ultimately proved to be an important one for optimizing the delivered image quality.

A filter exchange mechanism was also necessary. Since the focal plane obscures the science beam, any filter mechanism must have a profile not significantly larger than the focal plane itself. The solution adopted was to place each filter at the end of a long paddle which is driven to a position immediately in front of the field flattener lens, just before the focal plane. Eight identical mechanisms were constructed and installed around the perimeter of the cryostat. When retracted the filter paddles are out of the science beam completely. Each filter paddle contains four $60 \mathrm{~mm}$ square filters, with each filter covering one detector.

\subsection{Autoguider}

UKIRT has had a piezo-driven tip-tilt stage on the $f / 36$ secondary mirror since 1995 . With sampling rates of less than $100 \mathrm{~Hz}$ this does not significantly correct atmospheric seeing, but rather acts as a fast guider, removing guiding errors, vibration and windshake. WFCAM was designed so that it too would make use of the piezo stage, with its new $f / 9$ M2 mounting onto the same three flexure points. WFCAM includes a guide CCD in the focal plane, on-axis, between the four IR detectors.

A Marconi 47-10 device was selected as the guide CCD due its format and compact size. With a specification to read an arbitrary guide window out at $40 \mathrm{~Hz}$, rapid row clocking and small control loop latency was essential so a non-inverted mode version of the CCD was used. In actual operation, the autoguider runs at between 20 and $100 \mathrm{~Hz}$ depending on the brightness of the guide star. Great care in the design and testing of the autoguider housing (complete shielding, including use of a conductive window), was required to ensure negligible EMC interference between the CCD and IR detectors, which run asynchronously.

\subsection{Control and acquisition electronics}

The Hawaii-2 detectors can be read out in either one or eight channels per quadrant mode. Tests showed that early hopes of 
pixel rates of $1 \mathrm{Mhz}$ or greater were unrealistic. Yet the large pixel size results in exposure times as short as $5 \mathrm{~s}$. So a fast readout speed was required, and WFCAM was designed with a full 32-channel parallel readout per detector - 128 parallel readout channels for the entire focal plane. The entire focal plane is read out in $0.7 \mathrm{~s}$.

In order to keep the detector acquisition and control architecture as simple as possible, while maximising data throughput, WFCAM implements four identical, parallel acquisition and control paths - one for each detector. Each data channel consists of a detector, PCB, preamplifier, controller, and acquisition computer with disk and tape storage.

Each detector is mounted in its ZIF socket which is soldered to a flexi-rigid PCB containing passive detector protection components for a total of 32 outputs per PCB. The detector internal FET amplifiers (rather than external discrete components) are used due the limited space available on the PCBs.

Due to concerns over heat dissipation in the UKIRTWFCAM optical path, it was decided that the four controllers and their power supplies should be mounted under the primary mirror cell along with the rest of the Cassegrain electronics, requiring some $10 \mathrm{~m}$ of cables to reach the detectors. So low-power preamplifiers and clock-conditioners were designed and installed at the top of the cryostat, as close as possible to the detectors with differential outputs connecting to the controllers through the long cables. Tests in the lab showed that this configuration did not significantly degrade system noise, and performance on the telescope has proved to be satisfactory in this regard. Banding-removal algorithms have had to be implemented in the reduction pipeline to remove artifical image structure, though it does not appear that this results from the long WFCAM signal path, since the same interference effect is seen by other groups with conventional setups.

WFCAM was the first instrument to use four synchronised Astronomical Research Cameras Generation III controllers, each with 32-channels. Each controller includes four 8-channel video cards and 16-bit $500 \mathrm{kHz}$ A/D converters. A $250 \mathrm{MHz}$ duplex fibre link to the data acquisition computer was sufficient to meet the WFCAM requirements of $12 \mathrm{Megapixel} / \mathrm{s}$ sustained data rate, and $20 \mathrm{Megapixel} / \mathrm{s}$ in burst mode. Extra-stable resistors were also used in the controller construction to ensure high gain stability. For acceptable noise performance the clocks for all four detector channels had to be synchronised; this was done by designating one controller clock as master, and driving the other three controllers as slaves with a single digital interrupt line.

The acquisition computers (one per channel) are PCs running Linux with RTAI real-time extensions, each with 800 Gbytes of RAID-5 disk storage. Sufficient disk storage is therefore available at UKIRT to give a few weeks data storage capacity at normal data rates. A high disk failure rate at the $4200 \mathrm{~m}$ altitude of Mauna Kea was expected, and after a difficult beginning has now settled down to a rate of 1 disk failure every two months (out of 70 spinning disks). Each day the new data from the previous night is backed up to an ULTRIUM LTO-2 tape, which holds up to $380 \mathrm{~Gb}$. A copy of the compressed FITS files is then sent to the wide field astronomy unit at Cambridge University for processing.

\subsection{Alignment}

The optical design of WFCAM, which corrects aberrations out to large field angles, means that alignment is much more critical than for a normal instrument. For example, the usual M2 alignment degeneracy on a classical Cassegrain which allows an M2 decentre to be corrected by a tilt for zero central coma, does not apply for WFCAM. Everything must be coaxial and welladjusted to demanding tolerances.

The instrument itself mounts on the central steel plinth normally used for lifting the mirror during aluminizing ( $\mathrm{P}$ in Fig. 3), and its top surface is a key interface. Metrology of this surface reassuringly showed it to be very accurately parallel to the local primary mirror tangent plane. However, at the time of commissioning some uncertainty remained as to how well the plinth was centred relative to the axis of the primary. Eventually, on-sky tests showed any decentre to be small, with very little evidence of any coma in stellar images over the entire focal plane.

This alignment procedure was sufficient to give one arcsecond images over the full field of view at first light. Subsequent adjustments of both focal plane orthogonality and M2 decentre/tilt have improved the image quality further. On nights of good seeing the WFCAM image quality can be as low as $0.55^{\prime \prime}$ $F W H M$ at K. UKIRT itself is known to deliver lower quartile seeing of around 0.4 ". Combining this with a top-hat smoothing due to the $0.4^{\prime \prime}$ pixel size, it seems likely that the WFCAM optics are degrading the image by less than $0.2^{\prime \prime} F W H M$ - which is close to that predicted by toleranced optical modelling.

\section{WFCAM operations}

\subsection{Installation}

The installation of the $1400 \mathrm{~kg}$ WFCAM instrument onto the central plinth of the primary mirror by manouevring it through the telescope trusses is a challenging task which will be repeated many times during the lifetime of the instrument. The task is accomplished with a custom-modified hydraulic lift truck from Hyster USA. The truck, with precision fine-motion controls, allows the installation of WFCAM to be a straighforward and safe procedure.

\subsection{Observing with WFCAM}

WFCAM is a near-IR imager and as such is relatively straightforward to use. There are, however, some unusual aspects to its operation related to specific features of the opto-mechanical design.

The WFCAM detectors are spaced by $94 \%\left(774^{\prime \prime}\right)$ of the detector active area size (822") as shown in Fig. 2. So achieving a square imaged field on the sky (a tile) requires four exposures on a $798^{\prime \prime}$ square grid, resulting in $24^{\prime \prime}$ of overlap on common edges. These are nominal design values only, since engineering tolerances result in slightly different amounts of overlap along different edges.

Autoguiding in WFCAM is accomplished through a fixed CCD covering approximately 25 square arcmin of sky and operating at $40 \mathrm{~Hz}$. With these parameters a guide star of sufficient brightness will usually, but not always, be available by chance. So for efficiency, observations need to be prepared so that the telescope pointing position guarantees that a sufficiently bright guide star falls on the CCD; the guide star is selected in advance of observing and its position (and hence expected position on the CCD) is stored with the telescope and camera configuration. This means that a nominal pointing position may need to be tweaked a few arcminutes to ensure that a guide star is available, while the large camera field of view means this has a negligible scientific impact.

A greater problem comes when mapping, since an arbitrary change in the position of individual pointings to acquire a guide 
star might not leave any overlap with adjacent frames. This is solved in such cases by always stepping backward with respect to the mapping direction if a guide star is not available - that is, the overlap with the previous frame is increased until a guide star is found. Of course, this is all done off-line during observing preparation, using the US Naval Observatory or other guide star catalogues. At the telescope, pointing and guide star acquisition are then fully automatic.

The process of jittering is normally used in imaging to image the same field multiple times with slightly different offsets from which one may then obtain a sky frame via appropriate (e.g. median) filtering of the image stack. It also ensures that any astronomical object is imaged by a number of different pixels, improving photometry and allowing bad pixels to be rejected without loss of sky coverage. This procedure is in routine use on WFCAM together with microstepping - a special variant of jittering implemented to improve image sampling. By stepping a precise sub-fraction of an IR pixel size (e.g. 4.5 IR pixels) the resulting images may be recombined by simple interlacing, without requiring any resampling. WFCAM employs options for $2 \times$ $2(1 / 2$ pixel) and $3 \times 3$ (1/3 pixel microstepping).

\subsection{Data reduction pipeline and archive}

Data processing and storage are important considerations. With typical exposure times of 5 to $10 \mathrm{~s}$, and assuming a high instrument efficiency in survey mode, up to 200 Gbytes per night of data are typical. Furthermore, since data is taken in a semiautomatic mode, a data reduction pipeline had to be developed to work in real time at the telescope, allowing quality control by summit observers. Otherwise, given the level of instrument automation, it would be possible for many hours of bad data to be taken without the observer noticing. The pipeline performs a reasonably complete reduction online including sky subtraction and flatfielding, source extraction and catalogue creation with astrometry and photometry based on the many 2MASS sources found in every WFCAM field. Thus zeropoints are monitored to check on photometric conditions, as well as image FWHM to check the state of focus and seeing all in near real time. The summit pipeline runs routinely and, importantly, fast enough to keep up with the incoming data rate.

The main data reduction pipeline is run by the Cambridge University Astronomical Survey Unit (CASU). Raw data is sent to CASU from the Joint Astronomy Centre in Hawaii on ULTRIUM-I tapes. CASU are responsible for calibration and removal of instrumental signatures (Irwin et al. 2006). Source catalogues are extracted automatically and then archived along with reduced data by the Widefield Astronomy Unit (WFAU) at the University of Edinburgh. The WFCAM Science Archive (WSA - Hambly et al. 2006) is available at http://surveys.roe.ac.uk/wsa/ and may be searched using standard forms or freeform SQL (Structured Query Language).

\section{Instrument performance on sky}

Following an initial commissioning period WFCAM is now close to meeting all specifications. A summary of the current performance is shown in Table 3. The optical image quality of WFCAM is still uncertain because it is not possible to measure this independently of the telescope and seeing. Also, the overall system observing efficiency is slightly lower than expected; work is ongoing to improve this.
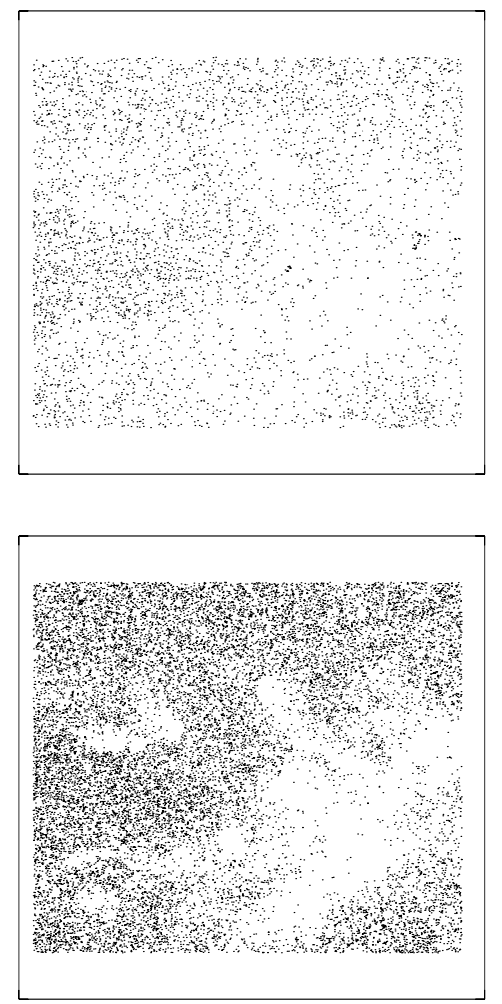

Fig. 4. $\rho$ Ophiuchi $1 \times 1$ degree field showing 2MASS $K$-band point source detections (top) and the same field as imaged in $K$ by WFCAM in $30 \mathrm{~min}$ (bottom).

As part of a guaranteed-time programme, WFCAM is being used to study the variability of very young obscured stars in the $\rho$ Ophiuchi and Orion Nebula clusters. The observing procedure in both cases is to execute observing blocks which image 0.8 square degree areas in $H$ and $K$, and to repeat this randomly over scales of days, weeks, months and yearly sampling periods. Each observing block takes approximately one hour and gives completeness limits for $H$ and $K$ of magnitudes 19 and 18 respectively. This type of project only really became possible in the IR with the advent of 2MASS (Carpenter et al. 2001), since historically the target field sizes have been far too large for normal IR imagers to cover in reasonable time. The WFCAM $\rho$ Ophiuchi observations go 3 mag fainter than the 2MASS catalogue and so (i) achieve $1 \%$ photometry at magnitude 15 , allowing small-amplitude variables to be discovered and (ii) allow large-amplitude variables to be discovered well into the brown dwarf regime. Figure 4 shows sources found by automatic extraction in the $\rho$ Ophiuchi core, by both WFCAM and 2MASS. Far more sources are evident in the WFCAM images, and in fact delineation of the cloud extinction structure can be seen down to small spatial scales.

Figure 5 shows two typical sources found in this sample, both at $K$ magnitude 15 . The non-variable source shows that photometry at the $1 \%$ stability level is being achieved, while the variable object shows the kinds of young star being targeted in this particular project.

The deepest survey which will be done with WFCAM is the UKIDSS Ultra-Deep Survey (UDS see Lawrence et al. 2006). The UDS will image an area of 0.77 sq. degs in the $J$ and $K$ filters to a depth of $K=23.0$, requiring 296 nights of UKIRT time over 7 years. The UDS is a single 

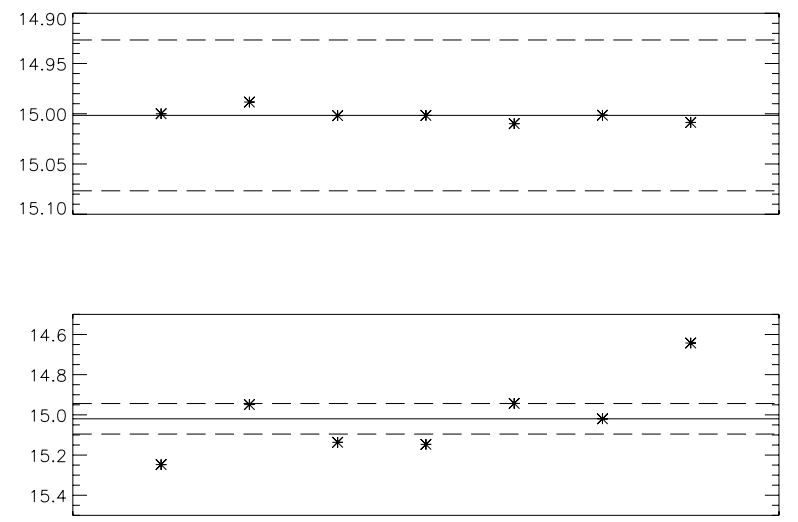

Fig. 5. Two $K$-band stellar magnitudes monitored over 7 epochs over several months in the $\rho$ Oph field. Dashed lines show $5 \sigma$ error bars on the mean. One star is constant (top) within errors while the other is variable (bottom).

filled-in square tile made from four WFCAM pointings. The target field is the Subaru and XMM-Newton Deep Survey Field (Sekiguchi et al. 2005). Figure 6 shows galaxy counts from the UDS Early Data Release as a function of $K$ magnitude, compared with various results from the literature (Saracco et al. 2001; Cristóbal-Hornillos et al. 2003; Iovino et al. 2005). An early discovery from this dataset is a significant number of massive galaxies at redshift $5<z<6$ (McLure et al. 2006).

Acknowledgements. The WFCAM project was funded by the UK Particle Physics and Astronomy Research Council.

\section{References}

Carpenter, J. M., Hillenbrand, L. A., \& Skrutskie, M. F. 2001, AJ, 121, 3160 Cristóbal-Hornillos, D., Balcells, M., Prieto, M., et al. 2003, ApJ, 595, 71 Dye, S., Warren, S. J., Hambly, N. C., et al. 2006, MNRAS, submitted Finger, G., Dorn, R., Meyer, M., et al. 2006, Proc. SPIE, 6276, in press Hambly, N., et al. 2006, in preparation

Henry, D. M., Casali, M. M., Montgomery, D., et al. 2003, Proc. SPIE, 4841, 63

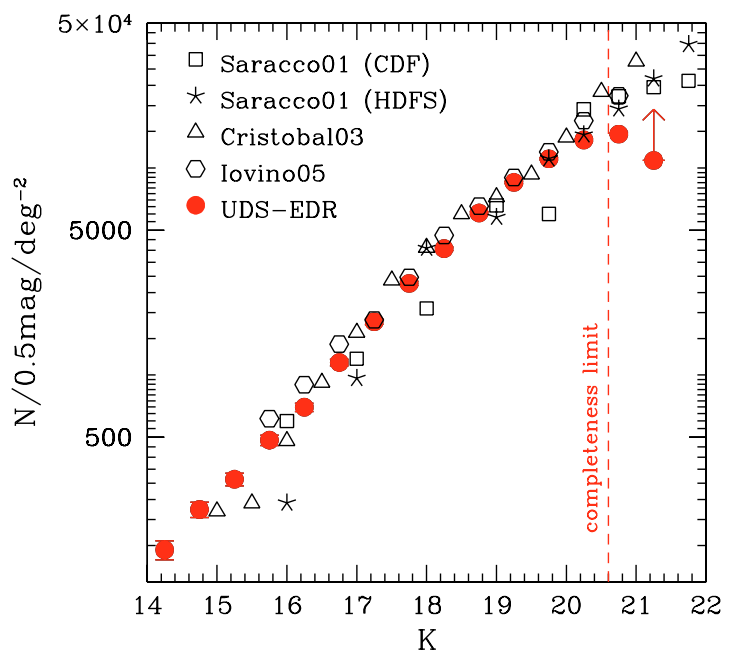

Fig. 6. Differential number counts as a function of $K$-band magnitude from the UKIDSS UDS Early Data Release, sampling a dynamic range of approximately 6 mag. At the completeness limit $(K=20.6)$ the majority of sources are distant galaxies, with median redshifts of $z \sim 1$. The final UDS survey will reach $K=23$.

Hewett, P. C., Warren, S. J., Leggett, S. K., \& Hodgkin, S. T. 2006, MNRAS, 367,454

Hillenbrand, L. A., Foster, J. B., Persson, S. E., \& Matthews, K. 2002, PASP, 114,708

Hirst, P., Casali, M. M., Adamson, A., Ives, D., \& Kerr, T. 2006, Proc. SPIE, 6269 , in press

Iovino, A., McCracken, H. J., Garilli, B., et al. 2005, A\&A, 442, 423

Irwin, M. J., et al. 2006, in preparation

Lawrence, A., Warren, S. J., Almaini, O., et al. 2006, MNRAS, in press [arXiv:astro-ph/0604426]

McLure, R. J., Cirasuolo, M., Dunlop, J. S., et al. 2006, MNRAS, submitted [arXiv: astro-ph/0606116]

Puget, P., Stadler, E., Doyon, R., et al. 2004, Proc. SPIE, 5492, 978

Saracco, P., Giallongo, E., Cristiani, S., et al. 2001, A\&A, 375, 1

Sekiguchi, K., Akiyama, M., Furusawa, H., et al. 2005, in Multiwavelength mapping of galaxy formation and evolution, ed. A. Renzini (Berlin: SpringerVerlag), 82

Skrutskie, M. F., Cutri, R. M., Stiening, R., et al. 2006, AJ, 131, 1163

Tokunaga, A. T., Simons, D. A., \& Vacca, W. D. 2002, PASP, 114, 180

York, D. G., Adelman, J., Anderson, J. E., et al. 2000, AJ, 120, 1579 